\title{
Überblick Verrechnungspreise
}

\author{
Roman Dawid
}

\subsection{Verrechnungspreise im Fokus der Öffentlichkeit}

Noch nie zuvor war die Besteuerung von grenzüberschreitend tätigen Unternehmen („MNEs“) so sehr im Fokus der Öffentlichkeit wie heute. Selbst investigative Journalisten haben die Verrechnungspreise als Thema für eine öffentlichkeitswirksame Story entdeckt. ${ }^{1}$ Die vermeintliche Steuervermeidung MNEs durch internationale Steuerplanung ist zu einem beständigen Thema in den Medien geworden. Im Fokus der Öffentlichkeit stehen Steuerplanungsmodelle weltweit bekannter Unternehmen, wie Apple, Google, Starbucks oder IKEA.

Als Reaktion hat die höchste Ebene der internationalen Politik, allen voran die G20 und internationale Institutionen wie die EU, die OECD sowie die Vereinten Nationen (UN) in den letzten Jahren umfangreiche Aktivitäten ergriffen und Maßnahmen verabschiedet, um eine mögliche Steuervermeidung durch Verrechnungspreise und andere internationale Steuerplanungsmodelle zu verhindern oder zumindest einzuschränken. Insbesondere haben die G20, die Gruppe der 20 größten Industrienationen, 2013 in Zusammenarbeit mit der OECD ein umfassendes Programm gegen Steuervermeidung und Gewinnverlagerung, „Base Erosion and Profit Shifiting“ kurz BEPS gestartet ${ }^{2}$. Die finalen Ergebnisse des BEPS Programms, das zahlreiche neue Regelungen zu Verrechnungspreisen enthält, wurden im November 2015 von den Regierungen der G20 verabschiedet.

${ }^{1}$ Vgl. Untersuchungen zu Verrechnungspreisthemen des International Consortium of Investigative Journalists (http://www.icij.org/).

${ }^{2}$ Vgl. OECD 2013.

\section{R. Dawid $(\bowtie)$}

PricewaterhouseCoopers AG Wirtschaftsprüfungsgesellschaft, Friedrich-Ebert-Anlage 35-37, 60327 Frankfurt am Main, Deutschland

E-Mail: roman.dawid@de.pwc.com

(C) Der/die Herausgeber bzw. der/die Autor(en) 2016

R. Dawid (Hrsg.), Verrechnungspreise, DOI 10.1007/978-3-658-09377-8_1 
Verrechnungspreise können für Unternehmen nicht nur zu substantiellen Steuernachzahlungen und möglicherweise Strafzahlungen sowie zu aufreibenden Auseinandersetzungen mit Finanzverwaltungen und Finanzgerichten führen. Sie sind darüber hinaus geeignet, Unternehmen in der Öffentlichkeit eine negative Reputation einzubringen. Verschiedene NGOs haben sich auf die Fahnen geschrieben, MNEs hinsichtlich Ihrer internationalen Steuerplanung zu beobachten und die Öffentlichkeit zu informieren. ${ }^{3}$

So wurden mehrfach MNEs an den medialen Pranger gestellt und Ihre Steuerplanung wurde als, wenn doch nicht illegal so doch zumindest als unmoralisch gebrandmarkt. Unter dem öffentlichen Druck sahen sich Unternehmen genötigt, zusätzlich zu ihren bisherigen Steuerzahlungen von sich aus zusätzliche Steuern zu zahlen, so beispielsweise geschehen im Fall Starbucks in Großbritannien, die zusätzliche Steuern in Höhe von 20 Mio. GBP für zwei Jahre gezahlt haben. ${ }^{4}$

\subsection{Worum geht es bei Verrechnungspreisen?}

Durch die Verrechnungspreise wird der zu versteuernde Gewinn eines international tätigen Unternehmens auf die beteiligten Länder aufgeteilt. Somit wird das Besteuerungsaufkommens eines MNEs zwischen den Jurisdiktionen verteilt, in denen das Unternehmen aktiv ist, d. h. in dem es Ableger, Tochtergesellschaften und/oder Betriebstätten unterhält. Das Vehikel für die Aufteilung sind die Verrechnungen zwischen den verschiedenen Konzerneinheiten, der Verrechnungspreise für Waren, Dienstleistungen und sonstigen Leistungen (z. B. Nutzungsrechte für Geistiges Eigentum) die zwischen den Einheiten des MNEs über die Grenze geliefert bzw. geleistet werden.

Das Folgende vereinfachte Beispiel soll dies verdeutlichen (Abb. 1.1).

Gesellschaft A der Unternehmensgruppe in Land A liefert Waren an Gesellschaft B der Unternehmensgruppe in Land B. Gesellschaft A produziert und verkauft die Waren grenzüberschreitend im Konzern. Gesellschaft B kauft im Konzern und verkauft an nicht verbundene, d. h. nicht zum Konzernverbund gehörende Kunden. B realisiert dabei einen Außenumsatz für die Unternehmensgruppe mit den externen Kunden. Der externe Preis der Waren, den Gesellschaft B mit den Kunden realisieren kann, entspricht 120. Es entstehen bei Unternehmen A Kosten in Höhe von 100. Kosten von B werden vereinfachend vernachlässigt. Der effektive Steuersatz entspricht 30\% in Land A und 10\% in Land B.

Hinsichtlich des Verrechnungspreises werden vereinfachend zwei extreme Fälle betrachtet: Im ersten Fall entspricht der Verrechnungspreis den Kosten von A in Höhe von 100. Damit erhält A gerade seine Kosten erstattet; der gesamte Konzerngewinn vor Steuern in Höhe von 20 fällt in Land B an und wird dort mit $10 \%$ beteuert. Im zweiten Fall entspricht der Verrechnungspreis dem externen Preis in Höhe von 120. Damit entsteht der gesamte Vorsteuergewinn in Land A und wird mit 30\% besteuert.

\footnotetext{
${ }^{3}$ Vgl. hierzu bspw. die Aktivitäten des Tax Justice Network (http://www.taxjustice.net/).

${ }^{4}$ Vgl. Neville, S. \& Treanor, J. Starbucks to pay $£ 20 \mathrm{~m}$ in tax over next two years after customer revolt.
} 


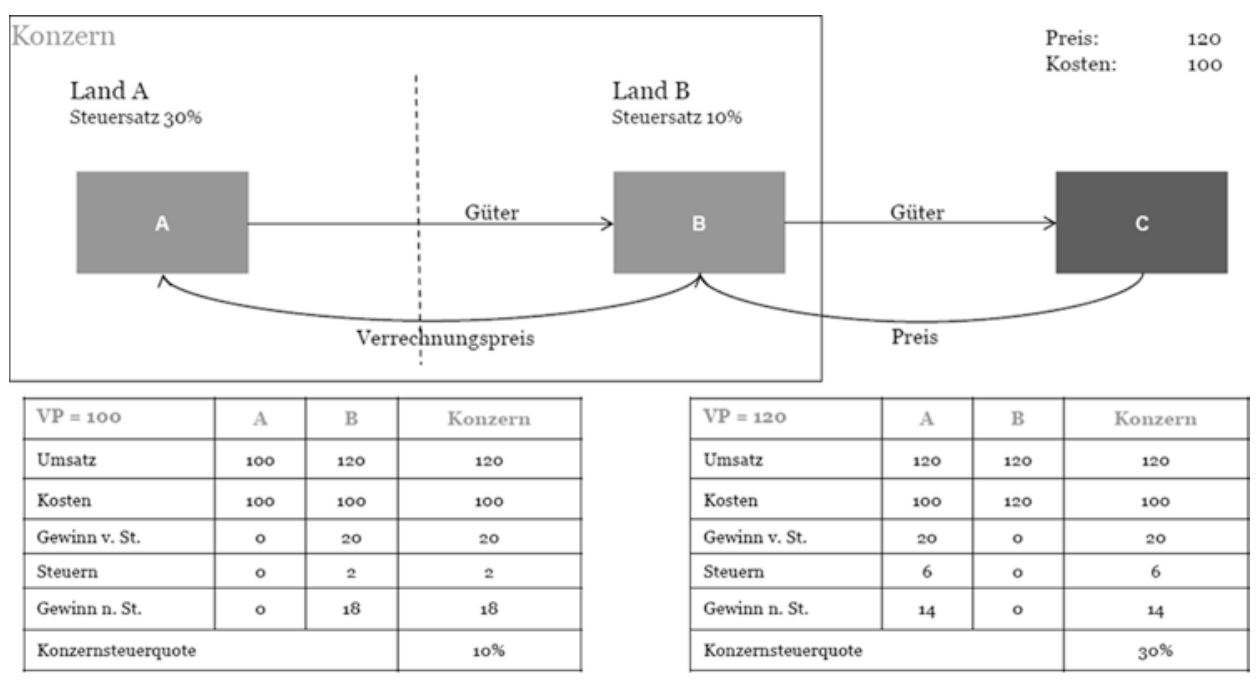

Abb. 1.1 Beispielhafte Darstellung von Konzernverrechnungspreisen

Im ersten Fall ist das Steueraufkommen deutlich niedriger und der Konzerngewinn nach Steuern somit höher (18) als im zweiten Fall (14). Der Gewinn vor Steuern ist in beiden Fällen gleich (20). Das Beispiel verdeutlicht, dass es sich aus Konzernsicht lohnen kann, einen möglichst niedrigen Verrechnungspreis festzulegen, um die Steuerquote des Konzerns zu minimieren (30\% versus 10\% Konzernsteuerquote). In der Praxis wird wahrscheinlich aufgrund der steuerlichen Regularien ein Preis zwischen den extremen Werten der betrachteten Fälle für den Verrechnungspreis festgelegt, so dass die Konzernsteuerquote zwischen 10 und $30 \%$ liegen wird.

Weil die Finanzverwaltungen in Land A und Land B jeweils ein Interesse haben, einen möglichst hohen Beitrag zum Steueraufkommen ihres Landes von dem Unternehmen zu erhalten, werden sie die Verrechnungspreise genau überprüfen und deren Angemessenheit möglicherweise in Frage stellen. Die meisten Länder haben für die Verrechnungspreise umfassende Vorschriften, die von den Unternehmen zu erfüllen sind und die vorgeben, worauf die Finanzverwaltungen bei der Prüfung der Verrechnungspreise zu achten haben. Kommt es bei der Prüfung in einem der beiden Länder zu einer Anpassung der Verrechnungspreise durch die entsprechende Finanzverwaltung, kann eine Doppelbesteuerung resultieren; d. h. insgesamt wird mehr Gewinn als der Konzerngewinn vor Steuern von 20 besteuert, d. h. mehr als die Unternehmensgruppe insgesamt tatsächlich erzielt. ${ }^{5}$

\footnotetext{
${ }^{5}$ Entsprechend spricht man von doppelter Nichtbesteuerung oder von „Weißen Einkünften“, wenn die steuerlichen Regelungen dazu führen, dass insgesamt in den betroffenen Ländern signifikant weniger als der Vorsteuergewinn von 20 der Besteuerung unterliegt. Dass eine Nichtbesteuerung resultiert, ist ein Kritikpunkt der Regierungen und NGOs. Das Ziel der Programme gegen Steuervermeidung, insbesondere des BEPS Programms ist es daher Nichtbesteuerung zu vermeiden.
} 
Die Vermeidung von Doppelbesteuerung ist das Ziel von bilateralen Abkommen zwischen den Ländern, den Doppelbesteuerungsabkommen, in denen u. a. Regeln für die Verrechnungspreise, deren Prüfung und Anpassung durch Finanzverwaltungen vereinbart werden. Dort sind in der Regel auch Lösungsmechanismen vereinbart, die die Unternehmen in Anspruch nehmen können, wenn es zu einer Doppelbesteuerung kommt. Die OECD sowie die UN haben als internationale Institutionen Musterabkommen entwickelt sowie umfassende Richtlinien für deren Umsetzung bzw. Anwendung als internationalen Standard veröffentlicht. Die überwiegende Anzahl der bilateralen Abkommen basiert auf dem Musterabkommen der OECD. Dieses enthält als wichtigstes internationales Leit-Prinzip für die Angemessenheit der Verrechnungspreise den sogenannten Fremdvergleichsgrundsatz. Entsprechend dem Fremdvergleichsprinzip dürfen die Finanzverwaltungen die Verrechnungspreise eines MNEs nur dann anpassen, wenn diese offensichtlich von dem abweichen, was unverbundene Parteien vereinbart hätten, die nicht in einen Konzern eingebunden sind.

\subsection{Wirtschaftliche Bedeutung von Verrechnungspreisen}

Weltweit hat der konzerninterne grenzüberschreitende Handel stark zugenommen. Die Globalisierung hat zu einem rasanten Anstieg von multinationalen Konzernen geführt. Der Anteil des grenzüberschreitenden Handels innerhalb von Unternehmen am gesamten Welthandel ist stark gestiegen: Der Anteil der Transaktionen innerhalb von MNEs am gesamten Welthandelsvolumen beträgt in etwa 80\%. ${ }^{6}$ Während die Anzahl der MNEs zwischen 1990 und 2010 mit einer durchschnittlichen Wachstumsrate von ca. $6 \%$ pro Jahr gewachsen ist, ist die Anzahl der Tochtergesellschaften von Konzernen im selben Zeitraum um ca. $9 \%$ gewachsen. $^{7}$

Obwohl zahlreiche empirische Studien zum möglichen Ausmaß der Steuervermeidung existieren, ist die Datenbasis zur tatsächlichen Steuervermeidung nach wie vor sehr unübersichtlich. Die OECD geht in ihrer jüngsten Veröffentlichung zur Messung von Steuervermeidung im Rahmen der finalen BEPS-Papiere davon aus, dass: ${ }^{8}$

- 4 bis $10 \%$ des globalen Steueraufkommens durch Steuervermeidung den Staaten nicht zur Verfügung steht. Dies würde laut OECD 100 bis 240 Mrd. USD jährlich entsprechen. Wirtschaftlich weniger entwickelte Länder sind hiervon systematisch weniger stark betroffen als wirtschaftlich entwickelte Länder;

\footnotetext{
${ }^{6}$ Vgl. United Nations Conference on Trade and Development, World Investment Report 2013: Global Value Chains: Investment and Trade for Development, S. 10.

${ }^{7}$ Vgl. United Nations Conference on Trade and Development, World Investment Reports 19912011.

${ }^{8}$ Vgl. OECD (2015). OECD presents outputs of OECD/G20 BEPS Project for discussion at G20 Finance Ministers meeting; OECD (2015), Measuring and Monitoring BEPS, Action 11.
} 
- die Renditen der Tochtergesellschaften von MNEs in Ländern mit niedrigem Steuersatz im Durchschnitt deutlich höher sind als die globale durchschnittliche Rendite der MNEs.

- die effektiven Steuerquoten großer MNEs im Durchschnitt signifikant niedriger sind als die effektiven Steuerquoten rein nationaler Unternehmen.

Die Schlussfolgerung der OECD ist, dass die Auswertung der Daten und Studien zu BEPS deutlich darauf hinweist, dass tatsächlich eine systematische Steuervermeidung durch die Steuerplanung der MNEs resultiert. Ob dies wirklich in größerem Umfang so ist, kann aus dem entsprechenden Papier zur Messung von BEPS kaum nachvollzogen werden, da die Datengrundlage größtenteils nicht zugänglich oder überprüfbar ist. Jedenfalls liefert das Ergebnis dieses Arbeitsbereich des BEPS-Programms der OECD und den Regierungen eine weitere Rechtfertigung für das gemeinsame Programm der G20 und der OECD gegen BEPS bzw. Steuervermeidung.

Bereits in der Vergangenheit, vor dem gemeinsamen BEPS-Programm, haben die Regierungen zahlreicher Länder bereits auf die Möglichkeit der Gewinnverlagerung mittels Verrechnungspreisen reagiert. In zahlreichen Ländern wurden umfangreiche nationale Regularien zu Dokumentationspflichten für Verrechnungspreise sowie resultierende Strafzuschlägen bei Nichtbefolgung dieser Vorschriften erlassen. Die nachfolgende Landkarte gibt einen Überblick in welchen Ländern Dokumentationspflichten für Verrechnungspreise gelten (Abb. 1.2). ${ }^{9}$

Es ist deutlich zu erkennen, dass es insbesondere in den wirtschaftlich entwickelten Regionen kaum ein Land gibt, das den Bereich der Verrechnungspreise nicht entsprechend geregelt hat. Darüber hinaus wurden in vielen nationalen Finanzverwaltungen, so auch in Deutschland, Kapazitäten von Betriebsprüfern aufgebaut, die gezielt internationale grenzüberschreitende Sachverhalte prüfen und hier insbesondere die Verrechnungspreise in den Fokus nehmen.

\subsection{Risiken aus Verrechnungspreisen für Unternehmen}

Im Zusammenhang mit den Verrechnungspreisen sind Unternehmen mit einem überaus komplexen Regelungsfeld konfrontiert: Einerseits sind, da es sich um grenzüberschreitende Transaktionen handelt, immer mindestens zwei Länder involviert. Damit bewegen sich die Unternehmen in der internationalen Sphäre des Steuerrechts, in der die Vereinbarungen zwischen den jeweiligen Ländern, die Doppelbesteuerungsabkommen sowie die Richtlinien und Interpretationshilfen zu diesen Abkommen internationaler Organisationen, insbesondere der OECD zu beachten sind.

\footnotetext{
${ }^{9}$ Einen aktualisierten Überblick über die Regularien in den verschiedenen Ländern gibt u. a. die PwC Transfer Pricing to Go App (http://www.pwc.com/gx/en/services/tax/transfer-pricing/appdownload.html).
} 


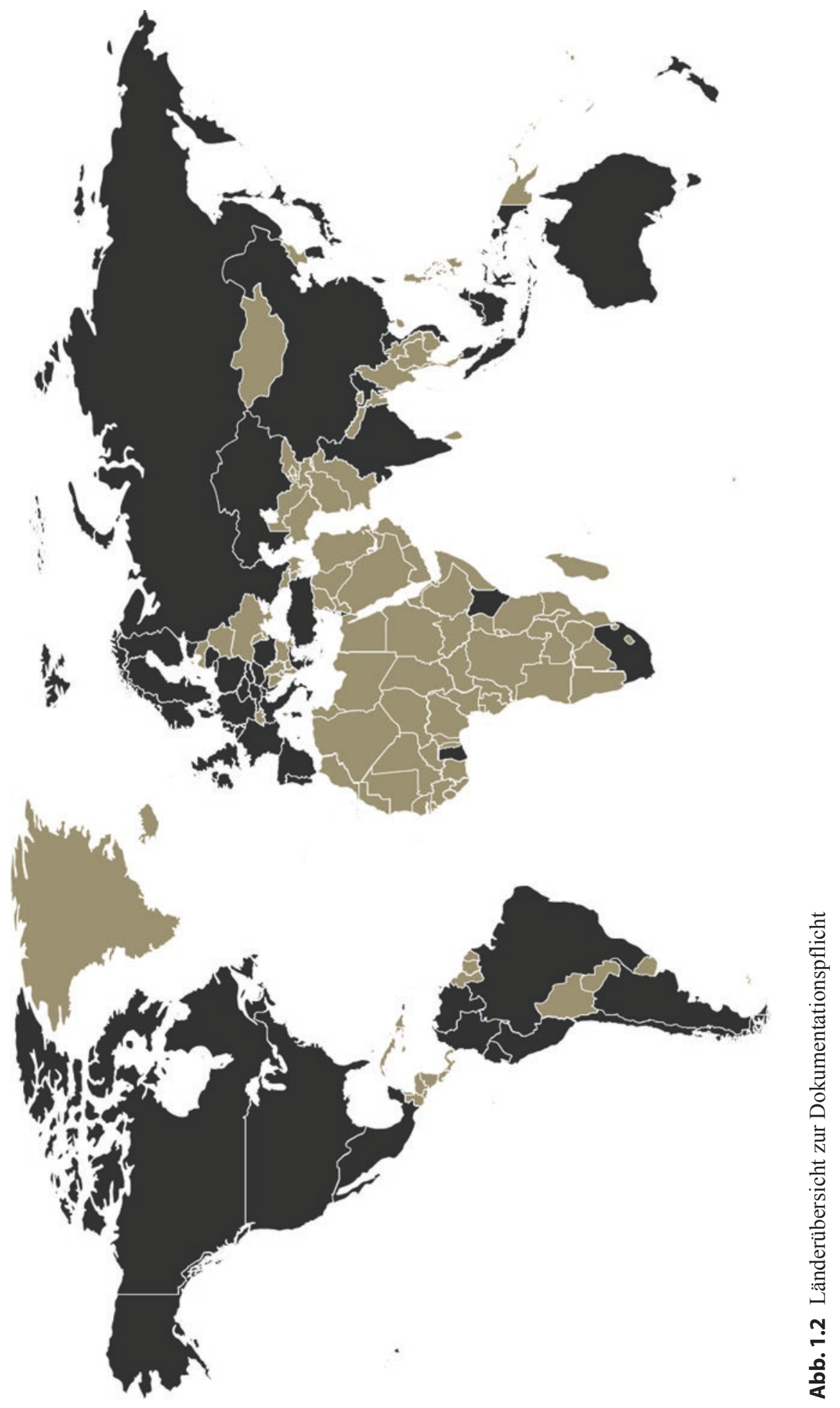


Andererseits haben zahlreiche Länder spezifische nationale Regelungen zum Thema Verrechnungspreise erlassen und zum Teil sind die Herangehensweisen der Finanzverwaltungen an dieses Prüfungsthema sehr unterschiedlich und oft ergebnisorientiert, d. h. mit dem klaren Ziel für den jeweiligen Fiskus ein Mehrergebnis zu erzielen. Dies kann für die multinationalen Unternehmen zu einer hohen Unsicherheit hinsichtlich der Akzeptanz der Verrechnungspreise durch die nationalen Finanzverwaltungen und damit der jeweiligen offenen Steuerpositionen in den verschiedenen Ländern führen. Selbst wenn ein MNE keine aggressive Steuerplanung betreibt und in erster Linie Rechtssicherheit anstrebt, kann das Unternehmen in der Regel nicht davon ausgehen, dass die Finanzverwaltungen den Verrechnungspreisansätzen folgen und keine Anpassungen durchführen werden.

Zudem ist der Bereich der Verrechnungspreise oft mit größeren Unsicherheiten, Graubereichen sowie subjektiven Annahmen verbunden. Der Fremdvergleichsgrundsatz, der das Leitprinzip für die Angemessenheit der Verrechnungspreise darstellt, ist zunächst eine Fiktion, die von der Realität der Entscheidungen innerhalb von Unternehmensgruppen abweicht: Der Fremdvergleichsgrundsatz geht von einer Transaktion zwischen unverbundenen Parteien aus, während in Konzernen in der Regel hierarchisch entschieden wird. Zudem ist er als solcher zunächst nicht konkretisiert und bedarf der Konkretisierung durch methodische Ansätze (z. B. Preisvergleich, Bruttomargenvergleich, Nettomargenvergleich) sowie empirischer Fremdvergleichsdaten zur tatsächlichen Bestimmung der Verrechnungspreise.

Eine Anleitung hinsichtlich der methodischen Ansätze zur Bestimmung fremdüblicher Preise wird in erster Linie durch die OECD Verrechnungspreis-Richtlinien gegeben ${ }^{10}$. Bei diesen handelt es sich um einen Verhandlungskompromiss zwischen den Finanzverwaltungen der beteiligten Länder unter Konsultation der interessierten Öffentlichkeit (Industrievertretungen, Berater etc.). Insofern ist die Anleitung oft vage und darum bemüht, genug Freiheitsgrade für den jeweiligen Einzelfall zu belassen. Diese Flexibilität ist oft im Sinne des Steuerpflichtigen, lässt aber ebensolche Flexibilität für die Argumentation der Finanzverwaltung. Dies führt zu signifikanten Unsicherheiten für die steuerpflichtigen Unternehmen im Bereich der Verrechnungspreise.

Hinzu kommt, dass es häufig schwierig ist, hinreichend vergleichbare Daten aus Transaktionen zwischen unverbundenen Parteien zu finden, die die Basis für einen Fremdvergleich bilden können. Entsprechende Fremdvergleichsdaten sind erforderlich, um auf Basis des jeweiligen methodischen Ansatzes eine numerische Bandbreite für die Verrechnungspreise abzuleiten. Ansonsten würde man sich ausschließlich im Bereich der Mutmaßung bzw. der Schätzung bewegen und es wäre noch schwieriger mit den Finanzverwaltungen zu einer Lösung zu kommen.

Aus diesem Grunde werden häufig Nettomargenmethoden angewendet, weil für diese Methode die Datenverfügbarkeit sehr gut und die Nachvollziehbarkeit der Daten relativ hoch ist. Allerdings ist die Skepsis der Finanzverwaltungen insbesondere in den Ländern mit relativ hohen effektiven Steuersätzen hinsichtlich der Nettomargenansätze groß und tendenziell im Laufe der Zeit gestiegen, weil die entsprechenden Ansätze oft nur einen begrenzten Anteil an dem gesamten Konzerngewinn ermöglichen und zudem eine

${ }^{10}$ Vgl. OECD 2010. 
Offenlegung des Gesamtgewinns aus den entsprechenden Geschäften vermieden werden kann. Dies spiegelt sich auch in den finalen BEPS-Papieren, die in Zukunft zumindest eine wesentlich genauere Analyse der Funktionen, Risiken sowie der werthaltigen immateriellen Wirtschaftsgüte („IWG“) als Voraussetzung für einen entsprechenden Ansatz erwarten. Jedenfalls kommt es in dem Bereich der Methoden für die Bestimmung der Verrechnungspreise schon heute häufig zu Diskussionen mit den Finanzverwaltungen. ${ }^{11}$

Zwei grundlegende Themen stehen in der Diskussion mit den Finanzverwaltungen im Rahmen von Betriebsprüfungen neben der Klärung des Sachverhalts im Zentrum:

\section{Waren die Verrechnungspreise angemessen, $d$. $h$. fremdüblich, und werden diese} dem Grunde und der Höhe nach von der Finanzverwaltung akzeptiert? Der internationale Standard ist hierbei wie oben ausgeführt der Fremdvergleichsgrundsatz. Die Diskussionen drehen sich dabei zumeist um die Angemessenheit des gewählten Verrechnungspreisansatzes, die richtige Anwendung der gängigen Verrechnungspreismethoden sowie die Auswahl und die Vergleichbarkeit der Vergleichstransaktionen. Diese Diskussion wird in vielen Fällen ergebnisorientiert geführt.

2. Wurden die Dokumentations- und Mitwirkungspflichten erfüllt? Hierbei geht es häufig um formale Kriterien, deren Nichterfüllung möglicherweise entsprechende Rechtsfolgen auslösen können. Beispielsweise ist es so, dass in Deutschland der Betriebsprüfer anhand der Verrechnungspreisdokumentation die Auswahl sowie die Anwendung des Verrechnungspreisansatzes nachvollziehen können soll. Dennoch sind die Anforderungen für eine „Unverwertbarkeit“" der Dokumentation sehr hoch. Die Rechtsfolgen einer nicht vorgelegten oder unverwertbaren Dokumentation können für den Steuerpflichtigen allerdings gravierend sein, da der Betriebsprüfer die Verrechnungspreise schätzen und dabei seine geschätzte Bandbreite zu Lasten des Steuerpflichtigen ausschöpfen kann. Zudem werden auf den resultierenden Anpassungsbetrag der Einkünfte Strafzuschläge von 5-10\% erhoben.

Im Bereich der Auseinandersetzung mit den Finanzverwaltungen werden aus Sicht der Steuerpflichtigen in Deutschland durchweg folgende Erfahrungen geteilt:

- Teilweise sind die Diskussionen mit den lokalen Finanzverwaltungen langwierig und aufwendig. Oft ist die Prüfstrategie der Finanzverwaltung undurchsichtig und aus Sicht des Steuerpflichtigen nicht nachvollziehbar. Das Ziel ist in solchen Fällen häufig, den Preis einer Einigung für den Steuerpflichtigen zu erhöhen. Erfahrungsgemäß und bestätigt durch entsprechende empirische Erhebungen werden viele Betriebsprüfungen im Bereich der Verrechnungspreise mit einem Kompromiss über substantielle Anpassungen der Verrechnungspreise und des Gewinns der lokalen Gesellschaft beendet. ${ }^{12}$ Substantielle Steuernachzahlungen und Zinszahlungen sind die Konsequenz. Weitere Konfliktlösungsmöglichkeiten, wie Finanzgerichtsverfahren oder Verständigungsverfahren, werden häufig nicht in Anspruch genommen.

\footnotetext{
${ }^{11}$ Vgl. PwC, Betriebsprüfung 2015, S. 42; siehe www.pwc.de/BP_2015.

12 Vgl. PwC, Betriebsprüfung 2015, S. 42.
} 
- Verfahren vor Finanzgerichten im Bereich der Verrechnungspreise sind ebenfalls sehr langwierig und können sich über Jahre hinziehen. In der Regel führen sie nicht zu einer Beseitigung der Doppelbesteuerung. Daher sind entsprechende Verfahren im Bereich der Verrechnungspreise auch eher selten zu beobachten und stellen tendenziell eine Ausnahmeoption dar.

- Internationale Verständigungsverfahren, bei denen die Finanzverwaltungen der beteiligten Länder über die jeweilige Verrechnungspreisfrage verhandeln, sind geeignet, die resultierende Doppelbesteuerung zu beseitigen. Allerdings sind solche Verfahren aufgrund der begrenzten Ressourcen der zuständigen Behörden (in Deutschland dem Bundeszentralamt für Steuern, BZSt) ebenfalls de facto relativ langwierig. Dies obwohl es mittlerweile zwischen vielen Ländern die Möglichkeit von Schiedsverfahren mit Einigungszwang nach einer bestimmten Frist gibt (beispielsweise innerhalb der EU durch die EU Schiedskonvention innerhalb von insgesamt drei Jahren).

Zusammenfassend können aus Sicht der betroffenen Unternehmen aus der Auseinandersetzung mit der Finanzverwaltung im Bereich der Verrechnungspreise insbesondere die folgenden Risiken resultieren:

- Substantielle Steuernachzahlungen und darauf anfallende Zinsen. Diese können für die Unternehmen eine Doppelbesteuerung bedeuten, wenn das entsprechende Einkommen bereits auf der/den anderen Seite/n der Transaktionen versteuert wurde;

- Strafzuschläge, z. B. wegen der Nichterfüllung der Dokumentations- und Mitwirkungspflichten;

- Langwierige, die Ressourcen des Unternehmens in Anspruch nehmende, Betriebsprüfungen;

- Ggf. langwierige Gerichtsverfahren und/ oder internationale Verständigungsverfahren zur Beseitigung einer resultierenden Doppelbesteuerung

\subsection{Was gilt es zu beachten?}

Aus den Ausführungen sollte deutlich geworden sein, dass die Verrechnungspreise bei international tätigen Unternehmen im Fokus der Finanzverwaltungen stehen, in deren Ländern das Unternehmen aktiv ist. Die Finanzverwaltungen haben in der Regel erkannt, dass die Verrechnungspreise einen Hebel bieten, mit dem sich das Steueraufkommen in der Betriebsprüfung dauerhaft erhöhen lässt - im Gegensatz zu steuerlichen Themen wie Rückstellungen, die nur zu einer rein zeitlichen Verschiebung der Besteuerung führen. Hinzu kommt, dass durch die öffentliche Diskussion die Steuerplanung grenzüberschreitender Unternehmen im Fokus steht, was den lokalen Finanzbehörden ggf. eine zusätzliche Begründung bzw. Rechtfertigung für umfangreiche Prüfungshandlungen und eine offensive Vorgehensweise in diesem Bereich liefert.

Die Ergebnisse des verabschiedeten BEPS-Programms ermöglichen den Finanzverwaltungen eine wesentlich breiter angelegte Dokumentation sowie Analysen im Bereich 
der Verrechnungspreise von den steuerpflichtigen Unternehmen einzufordern. Die Möglichkeiten für den Informationsaustausch zwischen den Finanzbehörden werden gleichzeitig ausgeweitet. Die Transparenz der Verrechnungspreisansätze für die lokalen Steuerbehörden wird hierdurch wesentlich erhöht. Während es in der Vergangenheit oft möglich und angemessen war, einseitig nur den lokalen Teil einer Transaktion darzustellen und mit einer einseitigen Methode zu verproben, wird es in Zukunft nicht immer einfach möglich sein, den Blick der Finanzverwaltung auf die Gesamtzusammenhänge im Konzern zu verhindern. Die Finanzverwaltungen werden durch den neuen OECD Dokumentationsansatz und das Country by Country Reporting wahrscheinlich einen umfassenden Blick auf den Konzern erhalten können (siehe hierzu die Ausführungen in Kap. 3).

Zudem werden die Argumentations- bzw. die Gestaltungsspielräume hinsichtlich der anzuwendenden Verrechnungspreisansätze eingeschränkt, so z. B. im Bereich der IWG, hinsichtlich der Risikoübernahme durch einzelne Konzerngesellschaften oder bei der Einrichtung eines Pools für die Finanzierung von Forschungs- und Entwicklungsaktivitäten. Die Finanzverwaltungen können noch umfangreichere Informationen zu der tatsächlichen ökonomischen Substanz, zu Kontroll- und Entscheidungsbefugnissen über bestimmte Aktivitäten einfordern. Außerdem können sie eher als bisher den vom Steuerpflichtigen als angemessen gewählten Verrechnungspreisansatz umqualifizieren, weil ihnen ein anderer Ansatz eher vereinbar mit dem Fremdvergleichsgrundsatz und den neuen Kriterien der OECD erscheint (vgl. hierzu die Ausführungen in den Kap. 4 und 5). Die Rechtssicherheit wird durch diese Änderungen für die steuerpflichtigen Unternehmen sicherlich nicht erhöht; eher steigt das Potenzial für Diskussionen mit den Finanzverwaltungen. Daher gilt es, sich auf die neuen Anforderungen aus dem BEPS-Programm einzustellen und die Substantiierung der gewählten Verrechnungspreisansätze in der Verrechnungspreisdokumentation anzupassen oder aber diese hinsichtlich ihrer Verteidigungsfähigkeit im Hinblick auf die neuen OECD-Ansätze zu überprüfen und ggf. zu revidieren.

Eine zumindest teilweise gute Nachricht ist sicherlich, dass die OECD für die Beurteilung der Verrechnungspreise einen international abgestimmten Dokumentationsansatz entwickelt hat, an dem sich bei der Überprüfung der Verrechnungspreise auch die Finanzverwaltungen orientieren sollten (siehe hierzu die Ausführungen in Kap. 3). Dieser neue OECD-Dokumentationsansatz ist einerseits relativ breit angelegt und enthält einige restriktivere und nachteilige Anforderungen aus Sicht der Steuerpflichtigen (z. B. das Country-By-Country-Reporting, regelmäßige zeitnahe Dokumentation, regelmäßiges z. T. jährliches Update der Fremdvergleichsstudien, Präferenz für lokale Vergleichsunternehmen sowie für ex-ante Preissetzung). Andererseits gibt es auch Bestandteile, die für die Steuerpflichtigen von Vorteil sind: so z. B. die Möglichkeit, die Dokumentation in Englisch einreichen zu können und die Definition von Wesentlichkeitsgrenzen für Transaktionen, die allerdings noch durch die lokalen Finanzverwaltungen zu definieren sind. Die Hoffnung besteht, dass dieser Ansatz in Zukunft geeignet ist, auch die meisten lokalen Anforderungen mit zu erfüllen, so dass die Steuerpflichtigen ihre weltweiten Dokumentationsanforderungen im Bereich Verrechnungspreise effizient erfüllen. In diesem Zusammenhang bleibt abzuwarten, wie die Länder die Regelungen in nationales 
Recht überführen werden, und es bleibt zu hoffen, dass es nicht zu allzu vielen signifikanten Abweichungen bei diesen Überführungen in nationale Regelungen kommen wird.

Um die Risiken im Bereich der Verrechnungspreise aus Sicht eines Steuerpflichtigen Unternehmens zu minimieren, empfiehlt sich für international aktive Unternehmen erfahrungsgemäß die Beachtung der folgenden Grundsätze:

- Das steuerpflichtige Unternehmen sollte so gut wie möglich den Fremdvergleichsgrundsatz und die OECD Verrechnungspreis-RL mit den Änderungen und Ergänzungen durch das OECD-BEPS Programm beachten und dies ausführlich dokumentieren. Die OECD-RL stellen eine Anleitung zur Erfüllung des Fremdvergleichsgrundsatzes dar. Die gestiegenen Anforderungen aus dem BEPS Programm werden verstärkt die Aufmerksamkeit der Finanzverwaltungen auf die Verrechnungspreise lenken.

- Auch wenn die Finanzverwaltungen oft eine Präferenz für die transaktionsbezogenen Standardmethoden (z. B. einen Preisvergleich) äußern, heißt dies nicht, dass die Ergebnisse aus solchen Ansätzen auch tatsächlich immer akzeptiert werden. Finanzverwaltungen gehen oft ergebnisorientiert vor. Nicht jede mögliche Fremdtransaktion führt zwangsläufig auch zu einem von den Behörden akzeptierten Ergebnis. Die Erwartung ist, dass mit den neuen OECD-Ansätzen im Bereich der Verrechnungspreise ganzheitliche gewinnorientierte Ansätze, wie die Gewinnaufteilungsmethode (Profit Split Methode) an Bedeutung gewinnen und von den Finanzverwaltungen zunehmend eingefordert werden.

- Der Steuerpflichtige sollte in jedem Fall die aktuellen und für die relevanten Länder spezifischen Dokumentations- und Mitwirkungsvorschriften beachten und erfüllen. In Zeiten von BEPS sollte er zudem regelmäßig die Umsetzung des OECD-Dokumentationsansatzes und der anderen BEPS Änderungen in nationales Recht beobachten. In der Regel kann die Beweislast durch die Erfüllung der Dokumentationspflichten auf die Finanzverwaltungen übertragen werden.

- Für den Fremdvergleich gibt es in der Regel Bandbreiten. Diese sind bei eingeschränkt vergleichbaren Transaktionen einzuengen. Der Steuerpflichtige hat die Möglichkeit die Bandbreiten zu nutzen, denn im Prinzip ist jeder Punkt in der (eingeengten) Bandbreite vereinbar mit dem Fremdvergleichsgrundsatz.

Internationale Unternehmen, die grenzüberschreitende Aktivitäten, eine Tochtergesellschaft und/oder Betriebstätten unterhalten und die relevanten Transaktionsgrenzen überschreiten (s. u. a. Kap. 2), sollten sich in jedem Fall mit dem Thema Verrechnungspreise auseinandersetzen, um keine bösen Überraschungen in zukünftigen Betriebsprüfungen zu erleben. Dies gilt auch für mittelständische Unternehmen, da es bislang nur in sehr begrenzten Maße Vereinfachungen und Wesentlichkeitsgrenzen für kleinere Unternehmen gibt. Oft gibt es durchaus die Möglichkeit zu pragmatischen Ansätzen. Allerdings sollte man den Dokumentationsansatz, insbesondere den Fremdvergleichsansatz nicht zu stark verkürzen, da dieser ggf. schwierige Diskussionen in der nächsten Betriebsprüfung vermeiden helfen kann. Zumindest kann ein guter Dokumentationsansatz wesentlich dazu beitragen, die Ausgangsposition für die kommende Betriebsprüfung auf eine solide Basis zu stellen und die Verteidigungsfähigkeit der Verrechnungspreisansätze in Betriebsprü- 
fungen und damit die Rechtssicherheit signifikant zu erhöhen. Erst Recht in den Zeiten des BEPS-Programms gilt, dass die proaktive Überprüfung der Verrechnungspreisansätze und deren Dokumentation erforderlich ist, um in den kommenden Betriebsprüfungen bestehen zu können (vgl. zur Vorgehensweise im Einzelnen Kap. 6).

\subsection{Aufbau dieses Buches}

Der Aufbau dieses Buches ist so gestaltet, dass die einzelnen Kapitel grundsätzlich unabhängig voneinander gelesen werden können. Der Leser hat somit die Möglichkeit nach konkretem Interesse direkt in ein bestimmtes Kapitel zu dem für ihn besonders relevanten Themenbereich einzusteigen. Da die einzelnen Kapitel dennoch einer inneren Logik folgend aufeinander aufbauen, empfehlen wir grundsätzlich die Lektüre des gesamten Buches.

Dieses Buch beginnt, nach dieser Einführung, mit einem Kapitel über die relevanten Grundlagen des deutschen Steuerrechts sowie der internationalen steuerrechtlichen Regularien im Zusammenhang mit Konzernverrechnungspreisen. Neu in diesem Kapitel ist ein Überblick über alle Maßnahmen des BEPS Programms. Dabei wird herausgearbeitet, welche Maßnahmen für die Verrechnungspreise von Bedeutung sind. Der Praktiker, der beispielsweise vorwiegend an einer Hilfe zur Erstellung einer Verrechnungspreisdokumentation oder Beispielen für typische Betriebsprüfungsfälle interessiert ist, kann dieses Kapitel zunächst überspringen. Sollten detailliertere Informationen über steuerrechtliche Zusammenhänge zu einem späteren Zeitpunkt erforderlich sein, können diese in diesem Kapitel entsprechend nachgelesen werden.

Kapitel 3 behandelt beispielhaft die praktische Erstellung einer Verrechnungspreisdokumentation. Hierzu werden die wesentlichen inhaltlichen Bausteine ausführlich erläutert. Die Besonderheiten des OECD BEPS Dokumentationsansatzes und dessen Implikationen für die Dokumentationspraxis werden dargestellt. Abschließend wird die Erstellung einer Verrechnungspreisdokumentation anhand eines konkreten Fallbeispiels in der Praxis dargelegt.

Kapitel 4 vertieft den Themenkomplex „,Funktions- und Risikoanalyse“, die sowohl für die analytische Bestimmung fremdüblicher Verrechnungspreise einschließlich der Auswahl einer steuerlich anwendbaren Verrechnungspreismethode essenziell ist, als auch einen elementaren Baustein jeder Verrechnungspreisdokumentation darstellt. Das Kapitel fasst die detaillierten Ausführungen beispielhaft anhand typischer „Funktions- und Risikoprofile" häufig auftretender Fallkonstellationen zusammen. Die Funktions- und Risikoanalyse ist das wesentliche Fundament für die Durchführung einer ökonomischen Analyse der Fremdüblichkeit der Verrechnungspreise. Die Neuerungen aus den finalen BEPS Papieren wurden eingearbeitet und die Konsequenzen dargestellt.

Kapitel 5 beschäftigt sich schließlich ausführlich mit den Verrechnungspreismethoden und der ökonomischen Analyse der Fremdüblichkeit der Verrechnungspreise. In diesem Kapitel werden die wesentlichen international akzeptierten Verrechnungspreismethoden dargestellt, deren Anwendbarkeit für verschiedene Fallkonstellationen diskutiert sowie die konkrete Durchführung des Fremdvergleichstests mit Hilfe empirischer 
Drittdaten erläutert. In diesem Bereich gibt es zahlreiche wesentliche Neuerungen insbesondere aus den Aktionspunkten 8 bis 10 des BEPS Programms, die in der Darstellung entsprechend berücksichtigt wurden. Darüber hinaus werden die Implikationen für die Verrechnungspreisansätze diskutiert.

Abschließend werden in Kap 6 typische Problemfelder in Betriebsprüfungen anhand konkreter Fallbeispiele dargestellt, einschließlich möglicher Lösungs- bzw. Verteidigungsansätze. Kapitel 6 diskutiert zudem grundlegende Fragen eines effizienten Managements von Betriebsprüfungen.

\section{Literatur}

Neville, S. \& Treanor, J. 2012. Starbucks to pay $£ 20 \mathrm{~m}$ in tax over next twoyears after customer revolt. In: The Guardian (http://www.theguardian.com/business/2012/dec/06/starbucks-to-pay$10 \mathrm{~m}$-corporation-tax)

OECD 2013. Addressing Base Erosion and Profit Shifting.

OECD 22. Juli, 2010. Transfer Pricing Guidelines for Multinational Enterprises and Tax Administrations. OECD Publishing (,OECD RL“)

OECD (2015). OECD presents outputs of OECD/G20 BEPS Project for discussion at G20 Finance Ministers meeting. http://www.oecd.org/ctp/oecd-presents-outputs-of-oecd-g20-beps-projectfor-discussion-at-g20-finance-ministers-meeting.htm

OECD (2015), Measuring and Monitoring BEPS, Action 11 - 2015 Final Report, OECD/G20 Base Erosion and Profit Shifting Project, OECD Publishing, Paris. http://dx.doi.org/10.1787/ 9789264241343-en („OECD (2015), Measuring and Monitoring BEPS, Action 11“)

PricewaterhouseCoopers AG Wirtschaftsprüfungsgesellschaft 2015, Betriebsprüfung 2015 („PwC, Betriebsprüfung 2015“)

United Nations Conference on Trade and Development. World Investment Reports 1991-2011 (http://unctad.org/wir)

United Nations Conference on Trade and Development, 2013. World Investment Report 2013: Global Value Chains: Investment and Trade for Development.

Open Access Dieses Kapitel wird unter der Creative Commons Namensnennung-Nicht kommerziell 2.5 International Lizenz (http://creativecommons.org/licenses/by-nc/2.5/deed.de) veröffentlicht, welche für nicht kommerzielle Zwecke die Nutzung, Verbreitung und Wiedergabe in jeglichem Medium und Format erlaubt, sofern Sie den/die ursprünglichen Autor(en), den Titel des Werks und die Quelle ordnungsgemäß nennen, einen Link zur Creative Commons Lizenz beifügen und im Falle einer Abwandlung durch einen entsprechenden Hinweis deutlich erkennbar machen, dass Änderungen vorgenommen wurden.

Die in diesem Kapitel enthaltenen Bilder und sonstiges Drittmaterial unterliegen ebenfalls der genannten Creative Commons Lizenz, sofern sich aus der Abbildungslegende nichts anderes ergibt. Sofern das betreffende Material nicht unter der genannten Creative Commons Lizenz steht und die betreffende Handlung nicht nach gesetzlichen Vorschriften erlaubt ist, ist auch für die oben aufgeführten nicht-kommerziellen Weiterverwendungen des Materials die Einwilligung des jeweiligen Rechteinhabers einzuholen.

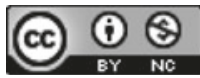

